\title{
Weak evidence for a benefit of Emdogain in the treatment of intrabony defects
}

\author{
Where patients have intrabony defects, is surgery with enamel matrix \\ derivative more effective than other treatments?
}

\begin{abstract}
Esposito M, Coulthard P, Worthington HV. Enamel matrix derivative (Emdogain ${ }^{\mathbb{R}}$ ) for periodontal tissue regeneration in intrabony defects (Cochrane Review). The Cochrane Library. 2003, Issue 2, Oxford: Update Software
\end{abstract}

Data sources Sources were the Cochrane Oral Health Group's Trials Register (to January 2003), the Cochrane Central Register of Controlled Trials, MEDLINE (1966 to January 2003) and EMBASE (1980 to January 2003). The International Journal of Periodontics and Restorative Dentistry, Journal of Clinical Periodontology, Journal of Dental Research, Journal of Periodontal Research, Journal of Periodontology and the bibliographies of papers and review articles were searched by hand. Authors, personal contacts and manufacturers were contacted in an attempt to identify unpublished or ongoing trials. There were no language restrictions.

Study selection The studies included were clinical randomised controlled trials (RCT) that considered enamel matrix derivative (EMD; Emdogain) [Biora, Malmö, Sweden] with at least 1 year followup.

Data extraction and synthesis Data were extracted by two reviewers independently, using specially designed data extraction forms. Results were expressed as random-effect models using weighted mean differences for continuous outcomes and relative risk for dichotomous outcomes with $95 \%$ confidence intervals $(\mathrm{Cl})$. Heterogeneity was investigated including both clinical and methodological factors.

Results No difference in tooth loss was observed. Meta-analysis of eight trials showed that EMD-treated sites displayed statistically significant probing attachment level (PAL) improvements (mean difference, $1.3 \mathrm{~mm} ; 95 \% \mathrm{Cl}: 0.8-1.8)$ and probing pocket depth (PPD) reduction (1 mm; 95\% Cl: $0.5-1.4$ ) compared with flap surgery. Six trials compared EMD with guided tissue regeneration (GTR), GTR showing a statistically significant reduction of PPD $(0.6 \mathrm{~mm})$ and increase of gingival recession $(0.5 \mathrm{~mm})$. No difference in postoperative infections was observed.

Conclusions EMD is able to significantly improve PAL levels $(1.3 \mathrm{~mm})$ and PPD reduction $(1 \mathrm{~mm})$ compared with flap surgery, but these results may not have a great clinical impact since it has not been shown that more periodontally compromised teeth could be saved. There was no evidence of clinically important differences between GTR and EMD.

\section{Commentary}

This well-conducted Cochrane review has tested the efficacy of EMD (Emdogain) in comparison with open-flap debridement, GTR and various bone-grafting procedures, over a follow-up period of at least 1 year. The authors conclude that EMD is able to significantly improve PAL levels and PPD reduction compared with flap surgery, but note that the results may not have great clinical impact.

The review amply demonstrates the heterogeneity of clinical-trial methodology, which is so characteristic of periodontal RCT.
Although the rigour of the Cochrane review methodology leaves little room for criticism, a few points may be noteworthy. The review identifies 10 RCT that fulfil the inclusion criteria. A quality assessment of these trials place only three trials in the high-quality category whereas five and two trials, respectively, are placed in the medium- and low-quality categories as regards the risk of bias. Nevertheless, all 10 RCT are included in the analyses.

The authors perform a metaregression analysis to assess the effect of various study characteristics - including trial quality - on the results and demonstrate that the mean effect of EMD on PAL change is $0.42 \mathrm{~mm}$ lower in the high-quality studies than in the lower-quality studies. Even so, the authors use absence of statistical significance to conclude that they were unable to explain the heterogeneity found between the studies. This, we submit, is a good illustration of the tyranny of the $P$ value. We suggest that the combination of trial quality $(0.42 \mathrm{~mm})$, place of conduct $(0.89 \mathrm{~mm})$ and use of antibiotics $(0.86 \mathrm{~mm})$ may explain the heterogeneity between studies.

The review shows that a statistical analysis was performed in all 10 trials to test the hypothesis of equality of the baseline characteristics of test and to control patients/defects. If baseline differences are observed in RCT, it is a matter of definition that they have arisen by chance. It does not take a statistical test to prove this point. The purpose of randomisation is to obtain a balanced distribution of known, as well as unknown, confounders. Such a balance may be difficult to achieve in small studies, however, such as most of the RCT included in this review. Rather than performing statistical tests, the distribution of known confounders across treatment arms should be described (obviously this is not possible for the unknown confounders). If important imbalances are noted (not a statistical hypothesis testing issue) some measure of confounder-control in the analysis of the data would be appropriate.

We agree with the authors of the review when they state that the CAL improvements attributable to EMD therapy may not have a great clinical impact. We question, however, whether the quality of the RCT available is sufficiently high to allow for a more definite estimation of the clinical efficacy of this intervention.

\section{Practice point}

- Currently, the evidence for a possible benefit of EMD in the treatment of intrabony defects is rather weak.

\section{Vibeke Baelum, Rodrigo Lopez}

Department of Community Oral Health and Paediatric Dentistry, Faculty of Health Sciences, University of Aarhus, Aarhus, Denmark

Evidence-Based Dentistry (2003) 4, 66.

doi:10.1038/sj.ebd.6400205 